\title{
High-resolution 3D MRI of mouse brain reveals small cerebral structures in vivo
}

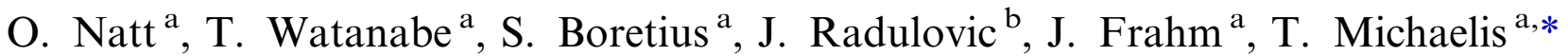 \\ ${ }^{a}$ Biomedizinische NMR Forschungs GmbH am Max-Planck-Institut für biophysikalische Chemie, 37070 Göttingen, Germany \\ ${ }^{\mathrm{b}}$ Molekulare Neuroendokrinologie, Max-Planck-Institut für experimentelle Medizin, Hermann-Rein-Straße 3, 37075 Göttingen, Germany
}

Received 22 March 2002; received in revised form 10 July 2002; accepted 11 July 2002

\begin{abstract}
This work demonstrates technical approaches to high-quality magnetic resonance imaging (MRI) of small structures of the mouse brain in vivo. It turns out that excellent soft-tissue contrast requires the reduction of partial volume effects by using $3 \mathrm{D}$ MRI at high (isotropic) resolution with linear voxel dimensions of about $100-150 \mu \mathrm{m}$. The long $T_{2} *$ relaxation times at relatively low magnetic fields $(2.35 \mathrm{~T})$ offer the benefit of a small receiver bandwidth (increased signal-to-noise) at a moderate echo time which together with the small voxel size avoids visual susceptibility artifacts. For measuring times of $1-1.5 \mathrm{~h}$ both * relaxation times at relatively low magnetic fields $(2.35 \mathrm{~T})$ offer the benefit of a small receiver bandwidth (increased signal-to-noise) at a moderate echo time which together with the small voxel size avoids visual susceptibility artifacts. For measuring times of $1-1.5 \mathrm{~h} \mathrm{both} T_{1}$-weighted (FLASH) and $T_{2}$-weighted (Fast Spin-Echo) 3D MRI acquisitions exhibit detailed anatomical insights in accordance with histological sections from a mouse brain atlas. Preliminary applications address the identification of neuroanatomical variations in different mouse strains and the use of $\mathrm{Mn}^{2+}$ as a $T_{1}$ contrast agent for neuroaxonal tracing of fiber tracts within the mouse visual pathway. (C) 2002 Elsevier Science B.V. All rights reserved.
\end{abstract}

Keywords: MRI; MR microscopy; Mouse brain; Mouse strain; Manganese; Contrast; Neuroaxonal tracing; Phenotyping

\section{Introduction}

During the past decade progress in neurogenetics has considerably facilitated the development of mutant mice. Since noninvasive magnetic resonance techniques provide structural, metabolic, and functional insights into the central nervous system in vivo and, furthermore, allow for repeated studies of the same animal, magnetic resonance imaging (MRI) and magnetic resonance spectroscopy are expected to become important research tools in system-oriented neurobiology with foreseeable contributions to functional genomics and considerable potential to bridge the gap to clinical applications.

\footnotetext{
* Corresponding author. Tel.: +49-551-201-1090; fax: +49-551201-1307

E-mail address: tmichae@gwdg.de (T. Michaelis).
}

Previous MRI studies of the brain of normal and/or mutant mice may be divided into two categories (for reviews, see Budinger et al., 1999; Jacobs et al., 1999b; Benveniste et al., 2000; Jacobs and Cherry, 2001; Kooy et al., 2001). First of all, post mortem imaging of excised specimen has reached near-microscopic spatial resolution as good as $15 \times 15 \times 15 \mu^{3}$ and is commonly performed at magnetic field strengths of $9.4 \mathrm{~T}$ or higher (Kornguth et al., 1994; Smith et al., 1994; Jacobs et al., 1999a). Although such studies yield geometrically undistorted three-dimensional representations of the brain or selected structures and thus extend histological reconstructions, they sacrifice the inherent noninvasiveness of MRI and, for example, exclude follow-up measurements during disease progression and for the evaluation of novel therapeutic interventions.

In contrast, the few in vivo MRI studies of mouse brain reported so far cover a wide range of voxel sizes and field strengths, for example $98 \times 156 \times 1500 \mu^{3}$ at 
$11.7 \mathrm{~T}$ (Dubowitz et al., 2000), $140 \times 140 \times 1200 \mu \mathrm{m}^{3}$ at $4.7 \mathrm{~T}$ (Hesselbarth et al., 1998), $156 \times 156 \times 1000 \mu \mathrm{m}^{3}$ at $14 \mathrm{~T}$ (Yang et al., 1999), $100 \times 200 \times 900 \mu^{3}$ at $2.39 \mathrm{~T}$ (Munasinghe et al., 1995), $78 \times 156 \times 343 \mu^{3}$ at $7 \mathrm{~T}$ (Kooy et al., 1999), and $150 \times 150 \times 150 \mu^{3}$ at $4.7 \mathrm{~T}$ (Xu et al., 1998). It is of interest to note, that most approaches emphasize a high in-plane resolution at the expense of a much poorer section thickness. While acceptable for larger animals and humans, the disregard of high spatial resolution in all three dimensions compromises the delineation of small structures (e.g., hippocampal formation) in the mouse brain (typical length $14 \mathrm{~mm}$, width $9 \mathrm{~mm}$, height $6 \mathrm{~mm}$ ) by significant partial volume effects.

The purpose of this study was (i) to develop $T_{1}$ - and $T_{2}$-weighted 3D MRI protocols for high-resolution studies of mouse brain in vivo which allow for the identification of small cerebral structures, (ii) to perform a direct comparison of anatomical structures with histological sections of $\mathrm{C} 57 \mathrm{BL} / 6 \mathrm{~J}$ mice as the most frequently used strain in gene knockout studies, and (iii) to examine the potential of these protocols for phenotyping of different mouse strains and in vivo tracing of axonal fiber connections after $\mathrm{Mn}^{2+}$ administration. A preliminary account has been given in abstract form (Natt et al., 2002).

\section{Materials and methods}

All studies were performed in accordance with German animal protection laws and approved by the responsible governmental authority. Young adult NMRI mice ( $n=18$, female), C57BL/6J mice $(n=4$, male), and BALB/c mice ( $n=4$, male) underwent MRI examinations at $2.35 \mathrm{~T}$ using a MRBR $4.7 / 400 \mathrm{~mm}$ magnet (Magnex Scientific, Abingdon, England) equipped with BGA20 gradients $\left(100 \mathrm{mT} \mathrm{m}^{-1}\right)$ driven by a DBX system (Bruker Biospin, Ettlingen, Germany).

For in vivo studies the animals were intubated and kept under halothane anesthesia $(1.0-1.5 \%$ halothane in 70:30 $\mathrm{N}_{2} \mathrm{O}: \mathrm{O}_{2}$ ). All animals were placed in a prone position with their heads firmly fixed in a purpose-built stereotactic device. Radiofrequency (RF) excitation and signal reception were accomplished with use of a Helmholtz coil (ø $100 \mathrm{~mm}$ ) and an elliptical surface coil (20 mm anterior-posterior, $12 \mathrm{~mm}$ left-right), respectively. The rectal body temperature was maintained constant at $37 \pm 1{ }^{\circ} \mathrm{C}$ using heated water blankets.

\section{1. $T_{1}$-weighted and $T_{2}$-weighted $3 D \mathrm{MRI}$}

To provide optimum $T_{1}$ contrast between gray and white matter as well as to facilitate the use of $T_{1}$ contrast agents and future applications such as angiography which depend on gradient-echo signals, $T_{1^{-}}$ weighted MRI was based on an RF spoiled 3D FLASH sequence. Even a relatively low receiver bandwidth of $12.5 \mathrm{kHz}$ resulted in only a moderate echo time of $7.6 \mathrm{~ms}$ which at $2.35 \mathrm{~T}$ poses no problems with regard to tissue susceptibility artifacts. In conjunction with the shortest possible repetition time of $17 \mathrm{~ms}$ the flip angle was adjusted to $25^{\circ}$ for optimum SNR and gray/white matter contrast.

Optimum $T_{2}$ contrast was achieved with use of an efficient fast spin-echo (FSE) sequence (flip angles: $90^{\circ}, 180^{\circ}$ ) with the mean echo time adjusted to $98 \mathrm{~ms}$. The resulting echo train allowed for the acquisition of 16 differently phase-encoded echoes with a receiver bandwidth of $16.7 \mathrm{kHz}$. To avoid compromising $T_{1}$ and $T_{2}$ signal alterations, a long repetition time of $3000 \mathrm{~ms}$ was chosen to reduce the effects from $T_{1}$ saturation.

Three dimensional data acquisitions were based on a field of view of $15 \times 15 \times 15 \mathrm{~mm}$ and in plane data matrices of $128 \times 96$, which after interpolation to $128 \times$ 128 (zero filling) yielded an image resolution of $117 \times$ $117 \mu \mathrm{m}^{2}$. To investigate the effect of section thickness, $T_{1}$-weighted 3D FLASH MRI was performed with 16 , 32,64 , and 96 phase encoding steps in the third direction providing section thicknesses of 938, 469, 234, and 156 $\mu \mathrm{m}$, respectively. The number of data accumulations for each section thickness was chosen to provide equal measuring times $(192,96,48,32$ accumulations for data sets acquired with 16, 32, 64, 96 phase encoding steps, respectively)

Fig. 1 shows mid-sagittal images which serve to define reference planes for intra- and interindividual comparisons independent of the orientation of the original $3 \mathrm{D}$ MRI data set. Since structural landmarks of the skull such as bregma and lambda are barely visible in MR images, the commonly used 'flat-skull' position is difficult to identify. As in human studies, the present work, therefore, employed the orientation of the anterior commissure (AC) to the posterior commissure (PC) to derive a suitable reference frame. Accordingly, a horizontal (coronal) reference plane was obtained by a $30^{\circ}\left(-60^{\circ}\right)$ rotation of the AC-PC plane as illustrated in Fig. 1.

\subsection{Retinal administration of manganese}

Mapping of retinal projections by $T_{1}$-weighted 3D MRI was accomplished $20 \mathrm{~h}$ after administration of a $0.5 \mu \mathrm{l}$ volume of a $120 \mathrm{mM}$ aqueous solution of $\mathrm{MnCl}_{2}$ (Sigma, Taufkirchen, Germany) into the vitreous body of the left eye. Intraocular injection of $\mathrm{Mn}^{2+}$ was performed over a 1 min period with a needle (17 mm length, $0.30 \mathrm{~mm}$ outer diameter) 

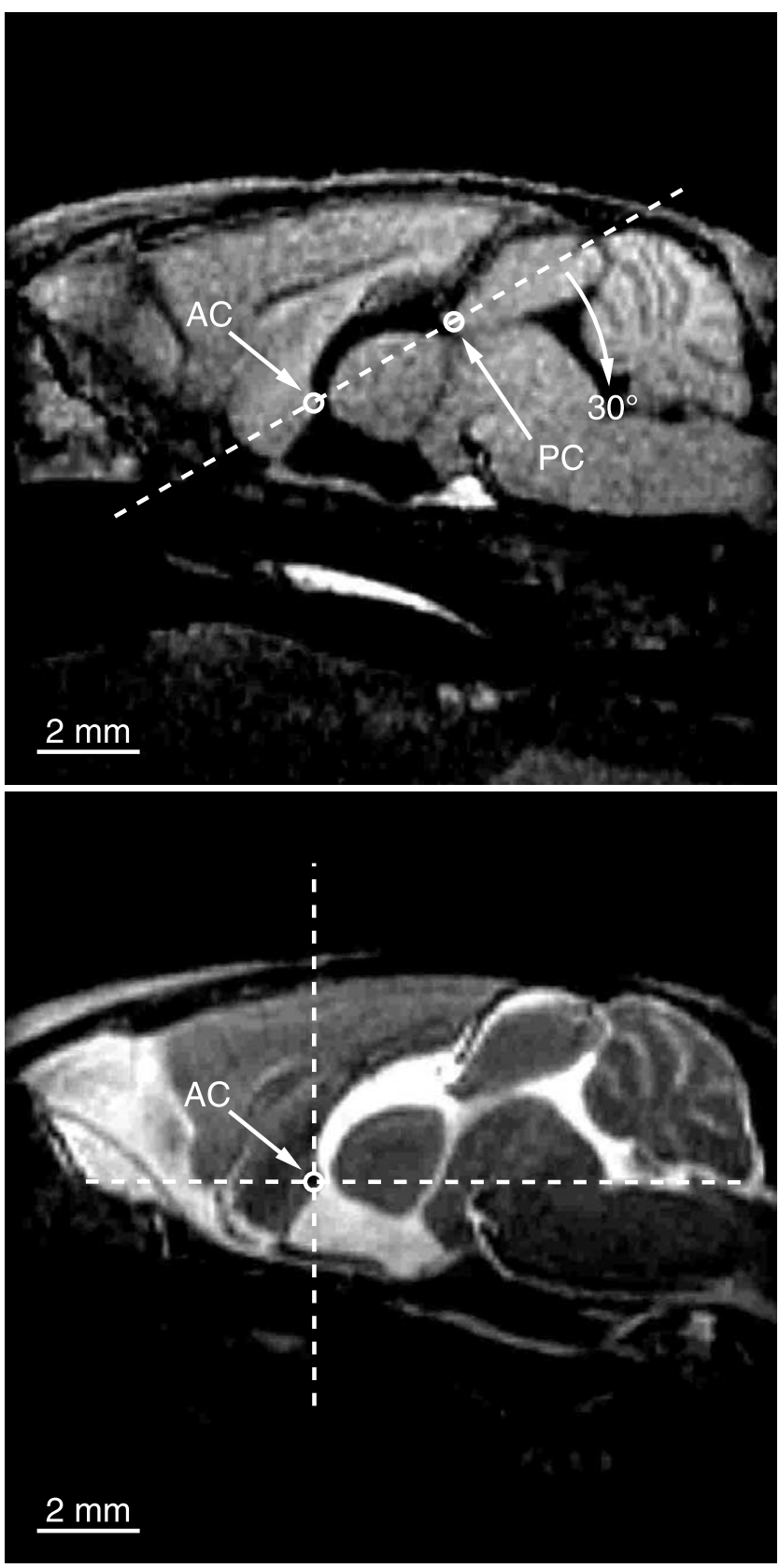

Fig. 1. (Top) $T_{1}$-weighted and (bottom) $T_{2}$-weighted high-resolution 3D MRI (mid-sagittal sections, for parameters see Table 1) of mouse brain in vivo $(\mathrm{C} 57 \mathrm{BL} / 6 \mathrm{~J})$ demonstrating reference planes for interindividual comparisons. After identification of the anterior and posterior commissure (AC, PC), horizontal and coronal reference planes are defined by a $30^{\circ}$ and $-60^{\circ}$ rotation of the $\mathrm{AC}-\mathrm{PC}$ plane around $\mathrm{AC}$, respectively.

connected via a polyethylene tube $(90 \mathrm{~mm}$ length, 0.28 $\mathrm{mm}$ inner diameter, Portex, UK) to a syringe. The needle was left in place for $10 \mathrm{~min}$ and then slowly withdrawn to minimize the loss of $\mathrm{MnCl}_{2}$ from leakage at the injection site. The procedure closely followed a protocol recently described for rats by Watanabe et al. (2001).

\section{Results and discussion}

\subsection{Partial volume effects and beyond}

The most enlightening aspect of the use of 3D MRI at suitable anatomical resolution is the unraveling of structural detail by removing partial volume effects. Fig. 2 demonstrates how unreasonably low resolution obscures contrast by merging signal intensity differences from neighboring though distinct structures. For image acquisitions with section thicknesses of $500 \mu \mathrm{m}$ or more (left two columns of Fig. 2), most of the tissue contrast is lost. While coarse structures of the mouse brain (e.g. cerebellum, midbrain, olfactory bulb) are readily visible in images at a section thickness of about $1000 \mu \mathrm{m}$, a section thickness of $500 \mu \mathrm{m}$ or less is necessary to resolve large white matter tracts such as corpus callosum, external capsule, and fimbria hippocampi. A further decrease of the section thickness to 234 or $156 \mu \mathrm{m}$ (right two columns of Fig. 2) reveals even smaller structures such as the hippocampal formation and the medial habenular nucleus. Note that the best soft-tissue contrast was obtained with the smallest section thickness. These findings favor the use of an isotropic spatial resolution for MRI of small brains. For mice such strategies lead to a desirable linear voxel dimension approaching $100 \mu \mathrm{m}$.

Of course, practical applications have to find a reasonable compromise between spatial resolution, SNR, and measurement time when aiming at in vivo studies. For the best resolution of $117 \times 156 \times 156 \mu \mathrm{m}^{3}$ ( $2.8 \mathrm{nl}$ voxel size without zero filling) acquired in this study, a SNR (calculated as the ratio of the image intensity in the region of interest and the standard deviation of the noise outside the mouse head) of 15-20 turned out to be sufficient to unveil anatomical details within the mouse brain. Taken the given experimental setup into account, i.e. the instrument hardware, animal arrangement, and RF coil design, such conditions were met for measuring times of $1-1.5 \mathrm{~h}$.

Based on these results, investigations demonstrating the applicability of 3D MRI for 'phenotyping of C57BL/ $6 \mathrm{~J}$ and $\mathrm{BALB} / \mathrm{c}$ mice' and 'mapping of retinal projections' were based on the parameters listed in Table 1.

\subsection{Phenotyping of $C 57 B L / 6 J$ and $B A L B / c$ mice}

Figs. 3 and 4 compare horizontal and coronal images from 3D MRI data sets of the brain of a C57BL/6J mouse with histological sections from a conventional anatomic atlas (Rosen et al., 2000). In close correspondence to the histology at $4.5 \times 4.5 \times 30 \mu \mathrm{m}^{3}$ resolution a large number of gray and white matter structures can be identified in both $T_{1^{-}}$and $T_{2}$-weighted images (see captions). 

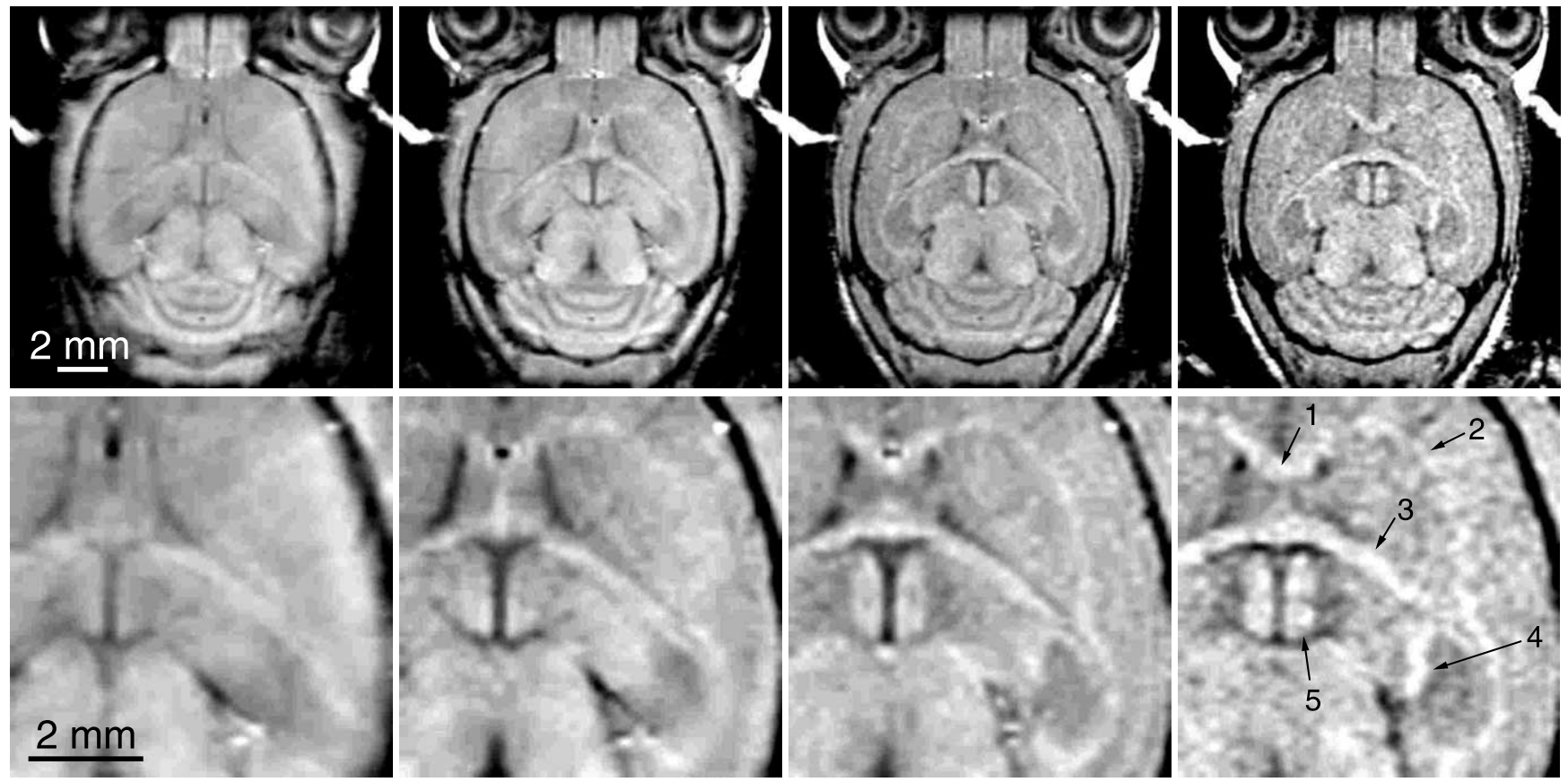

Fig. 2. (Top) $T_{1}$-weighted 3D MRI (horizontal sections) of the mouse brain in vivo (NMRI) demonstrating enhanced soft-tissue contrast with increasing spatial resolution and concomitant reduction of partial volume effects. From left to right, the voxel size decreases from $117 \times 117 \times 938$ $\mu \mathrm{m}^{3}(12.8 \mathrm{nl})$, to $117 \times 117 \times 469 \mathrm{~m}^{3}(6.4 \mathrm{nl}), 117 \times 117 \times 234 \mu \mathrm{m}^{3}(3.2 \mathrm{nl})$, and $117 \times 117 \times 156 \mu \mathrm{m}^{3}(2.1 \mathrm{nl})$. (Bottom) Magnified views of the images shown above demonstrate the (1) corpus callosum, (2) external capsule, (3) fimbria hippocampi, (4) hippocampal formation, (5) medial habenular nucleus.

Table 1

Parameters for $T_{1}$-weighted (FLASH) and $T_{2}$-weighted (FSE) highresolution MRI

\begin{tabular}{lll}
\hline & 3D FLASH & 3D FSE \\
\hline Repetition time & $17 \mathrm{~ms}$ & $3000 \mathrm{~ms}$ \\
Echo time & $7.6 \mathrm{~ms}$ & $98 \mathrm{~ms}($ mean) \\
Receiver bandwidth & $12.5 \mathrm{kHz}$ & $16.7 \mathrm{kHz}$ \\
Echoes & 1 & 16 \\
Flip angle & $25^{\circ}$ & $90^{\circ}, 180^{\circ}$ \\
Field of view & $15 \times 15 \times 15 \mathrm{~mm}^{3}$ & $15 \times 15 \times 15 \mathrm{~mm}^{3}$ \\
Acquisition matrix & $128 \times 96 \times 96$ & $128 \times 96 \times 96$ \\
Image resolution & $117 \times 156 \times 156 \mu \mathrm{m}^{3}$ & $117 \times 156 \times 156 \mu \mathrm{m}^{3}$ \\
Zero filling to & $117 \times 117 \times 117 \mu \mathrm{m}^{3}$ & $117 \times 117 \times 117 \mu \mathrm{m}^{3}$ \\
Accumulations & 32 & 2 \\
Measuring time & $84 \mathrm{~min}$ & $58 \mathrm{~min}$ \\
\hline
\end{tabular}

In general, the present results show a slight tendency in favor of $T_{1}$-weighted gradient-echo MRI sequences for the analysis of the normal neuroanatomy. However, the usefulness and sensitivity of $T_{2}$-weighted sequences for the detection of pathologic alterations is well appreciated. Here, major white matter tracts such as the external capsule, AC, and fimbria can be well differentiated in both $T_{1^{-}}$and $T_{2}$-weighted MRI, whereas $T_{1}$-weighted $3 \mathrm{D}$ FLASH appears to be more sensitive for a delineation of the hippocampal formation. The utility of $T_{1}$-weighted MRI is also supported by the images shown in Fig. 5 depicting neuroanatomical variations in sagittal, horizontal, and coronal sections of two different mouse strains. The images in the left column reveal the much larger ventricular spaces of the C57BL/6J mouse in comparison with the BALB/c mouse. Corresponding differences are most pronounced in the ventricles of the forebrain.

\subsection{Mapping of retinal projections}

Despite measuring times of $1-1.5 \mathrm{~h}$, high-resolution 3D MRI acquisitions allow for dynamic studies of slow functional processes such as the neuronal uptake and axonal transport of $\mathrm{Mn}^{2+}$ as an analogue to $\mathrm{Ca}^{2+}$. As demonstrated in Fig. 6, this concept has been exploited for mapping the retinal projections $20 \mathrm{~h}$ after intraocular injection of $\mathrm{Mn}^{2+}$ into the left eye. The combination of $\mathrm{Mn}^{2+}$-induced signal enhancement and $T_{1^{-}}$ weighted 3D FLASH results in a functional delineation of axonal connectivities within the visual pathway of a living mouse. The selected sections cover the retinal 

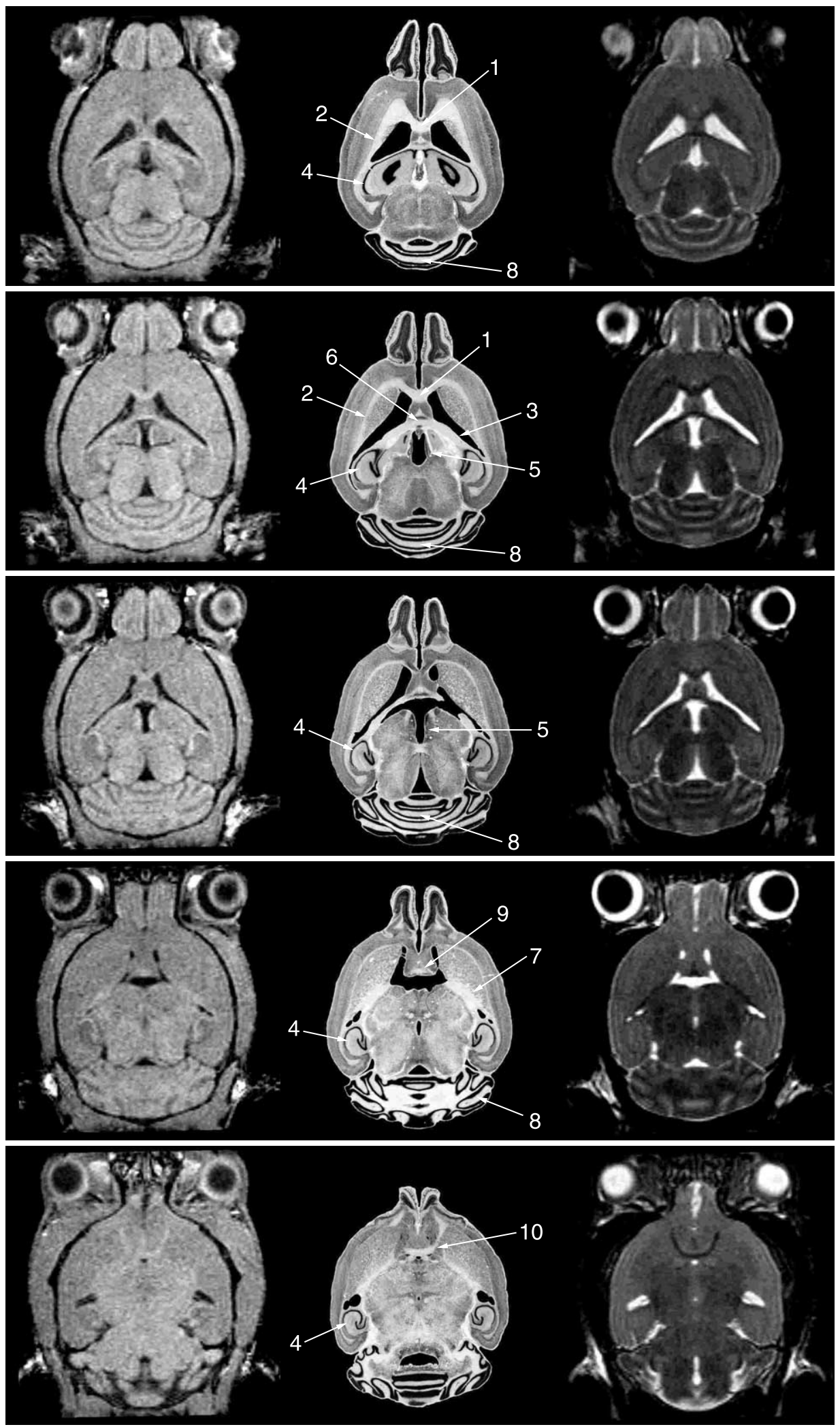

Fig. 3. (Left) $T_{1}$-weighted and (right) $T_{2}$-weighted high-resolution 3D MRI (horizontal sections, for parameters see Table 1) of mouse brain in vivo (C57BL/6J) in comparision with (middle) histological sections (Reproduced with permission from Rosen et al., 2000). From top to bottom the sections are 2.2, 1.9, 1.7, 1.1 and $0.0 \mathrm{~mm}$ dorsal to the reference plane in Fig. 1. The following structures are well delineated: (1) corpus callosum, (2) external capsule, (3) fimbria hippocampi, (4) hippocampal formation, (5) medial habenular nucleus, (6) ventral hippocampal commissure, (7) internal capsule, (8) cerebellar sulci, (9) fornix, and (10) anterior commissure. 

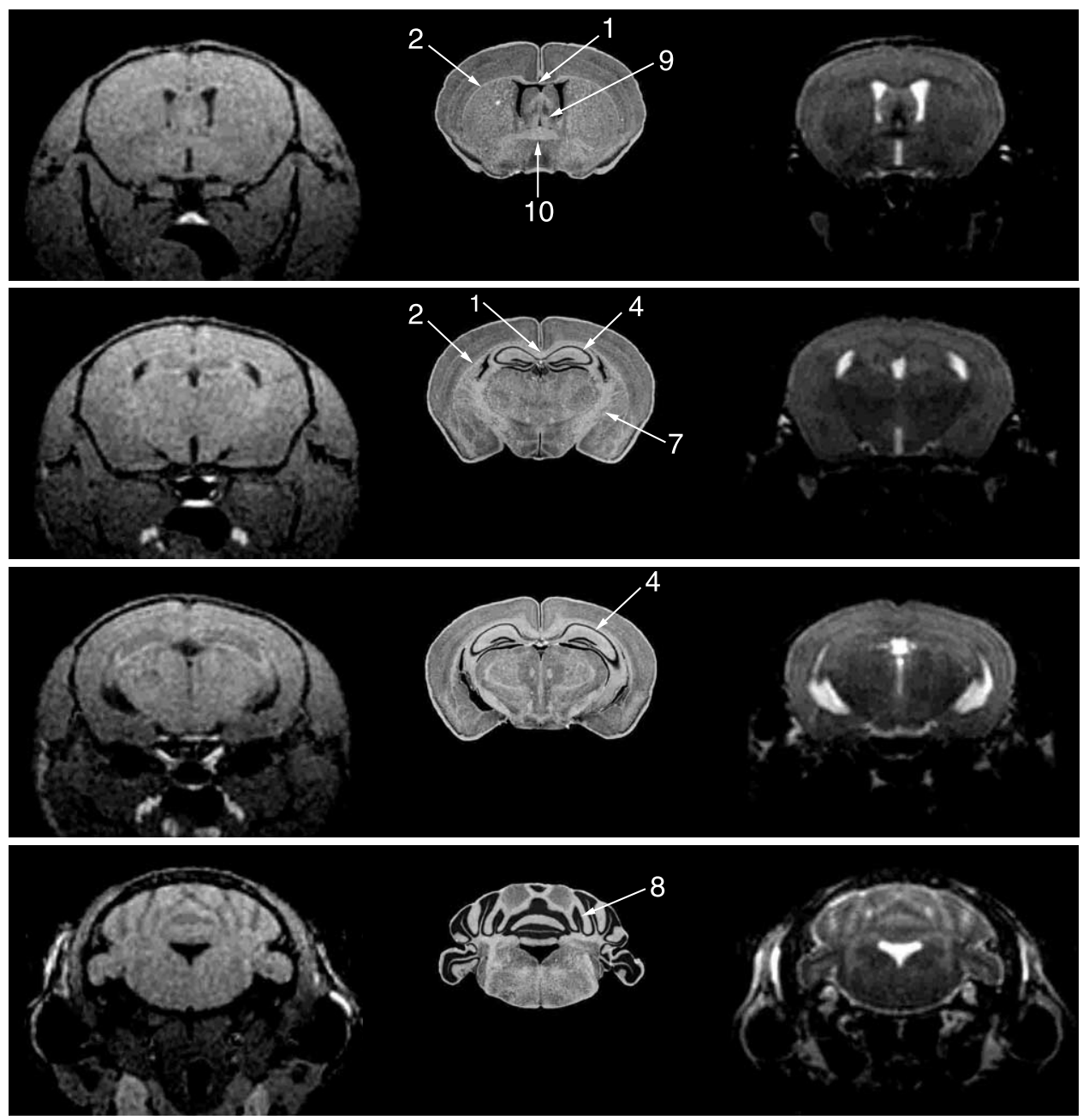

Fig. 4. (Left) $T_{1}$-weighted and (right) $T_{2}$-weighted high-resolution 3D MRI (coronal sections, same data set as in Fig. 3) of mouse brain in vivo (C57BL/6J) in comparison with (middle) histological sections (Reproduced with permission from Rosen et al., 2000). From top to bottom the sections are $0.0,1.7,2.5$, and $5.7 \mathrm{~mm}$ posterior to the reference plane shown in Fig. 1. The following structures are well delineated: (1) corpus callosum, (2) external capsule, (4) hippocampal formation, (7) internal capsule, (8) cerebellar sulci, (9) fornix, and (10) anterior commissure.

projections from the left retina up to the right superior colliculus.

\section{Conclusion}

It has been shown that high-resolution 3D MRI studies of mouse brain in vivo yield excellent SNR and soft-tissue contrast. In this context, the use of isotropic or near-isotropic spatial resolution with linear voxel dimensions of about $100-150 \mu \mathrm{m}$ (corresponding to volumes of 1.0-3.4 nl) turns out to be a prerequisite for minimizing partial volume effects and thus enhancing the contrast of the small anatomical structures of mouse brain. The surprisingly good performance of the low field, in particular for 3D gradient-echo MRI, stems from the fact that $T_{2}{ }^{*}$ relaxation times are much longer at low than at high magnetic fields. This allows for the use of small receiver bandwidths at moderate echo times which, together with the small voxel size, have the additional advantage of avoiding visible susceptibility artifacts.* relaxation times are much longer at low than at high magnetic fields. This allows for the use of small receiver bandwidths at moderate echo times which, together with the small voxel size, have the additional advantage of avoiding visible susceptibility artifacts.

In summary, the techniques presented here provide access to excellent anatomical details of mouse brain as well as of functional aspects with the option for repeated studies of the same animal. The experimental findings suggest that - independent of the actual field strength 3D MRI at high spatial resolution bears considerable potential for in vivo phenotyping and the evaluation of therapies in mutant mice. 

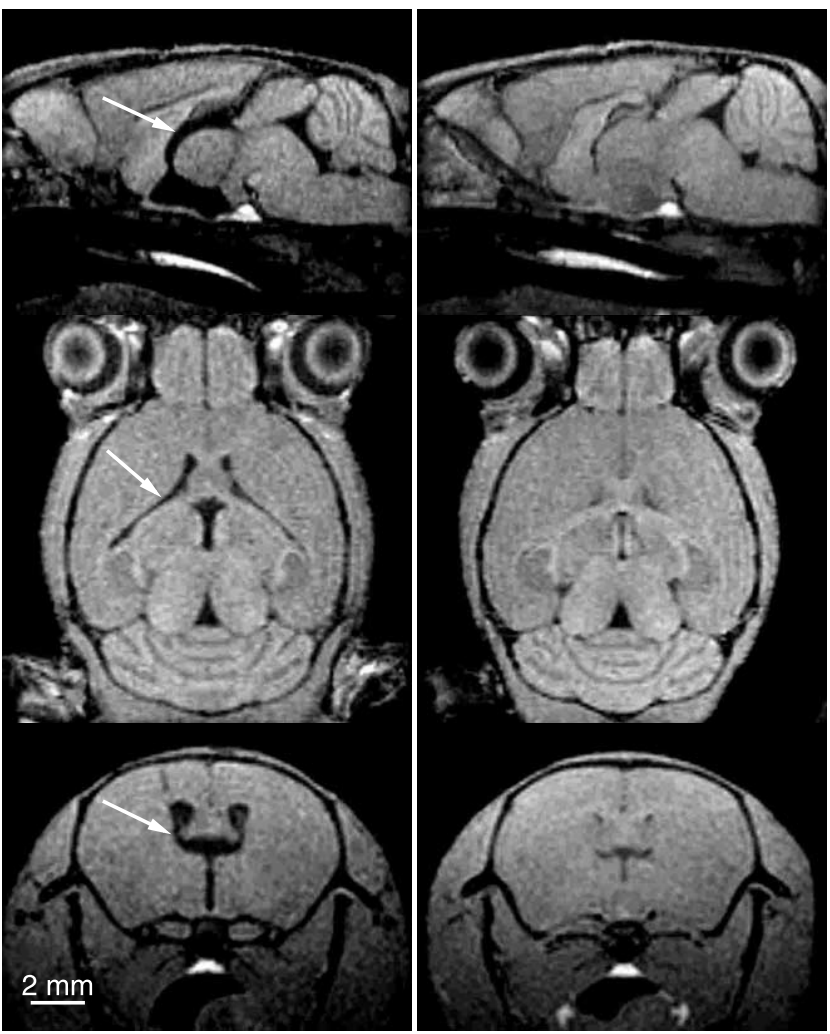

Fig. 5. High-resolution 3D FLASH MRI (for parameters see Table 1) of the brain of (left) a C57BL/6J and (right) a BALB/c mouse in vivo demonstrating structural variations in different strains such as larger ventricles in the forebrain (arrows). The images refer to (top) a midsagittal position, (middle) a horizontal section $1.5 \mathrm{~mm}$ shifted dorsal to the reference plane in Fig. 1, and (bottom) a coronal section $0.3 \mathrm{~mm}$ posterior to the reference plane.

\section{References}

Benveniste H, Kim K, Zhang L, Johnson GA. Magnetic resonance microscopy of the C57BL mouse brain. Neuroimage 2000;11:60111 .

Budinger TF, Benaron DA, Koretsky AP. Imaging transgenic animals. Annu Rev Biomed Eng 1999;1:611-48.

Dubowitz DJ, Tyszka JM, Sewry CA, Moats RA, Scadeng M, Dubowitz V. High resolution magnetic resonance imaging of the brain in the dy/dy mouse with merosin-deficient congenital muscular dystrophy. Neuromuscul Disord 2000;10:292-8.

Hesselbarth D, Franke C, Hata R, Brinker G, Hoehn-Berlage M. High resolution MRI and MRS: a feasibility study for the investigation of focal cerebral ischemia in mice. NMR Biomed 1998;11:423-9.

Jacobs RE, Cherry SR. Complementary emerging techniques: highresolution PET and MRI. Curr Opin Neurobiol 2001;11:621-9.

Jacobs RE, Ahrens ET, Dickinson ME, Laidlaw D. Towards a microMRI atlas of mouse development. Comput Med Imaging Graph 1999a;23:15-24.

Jacobs RE, Ahrens ET, Meade TJ, Fraser SE. Looking deeper into vertebrate development. Trends Cell Biol 1999b;9:73-6.

Kooy RF, Reyniers E, Verhoye M, Sijbers J, Bakker CE, Oostra BA, Willems PJ, van der Linden A. Neuroanatomy of the fragile $X$ knockout mouse brain studied using in vivo high resolution magnetic resonance imaging. Eur J Hum Genet 1999;7:526-32.
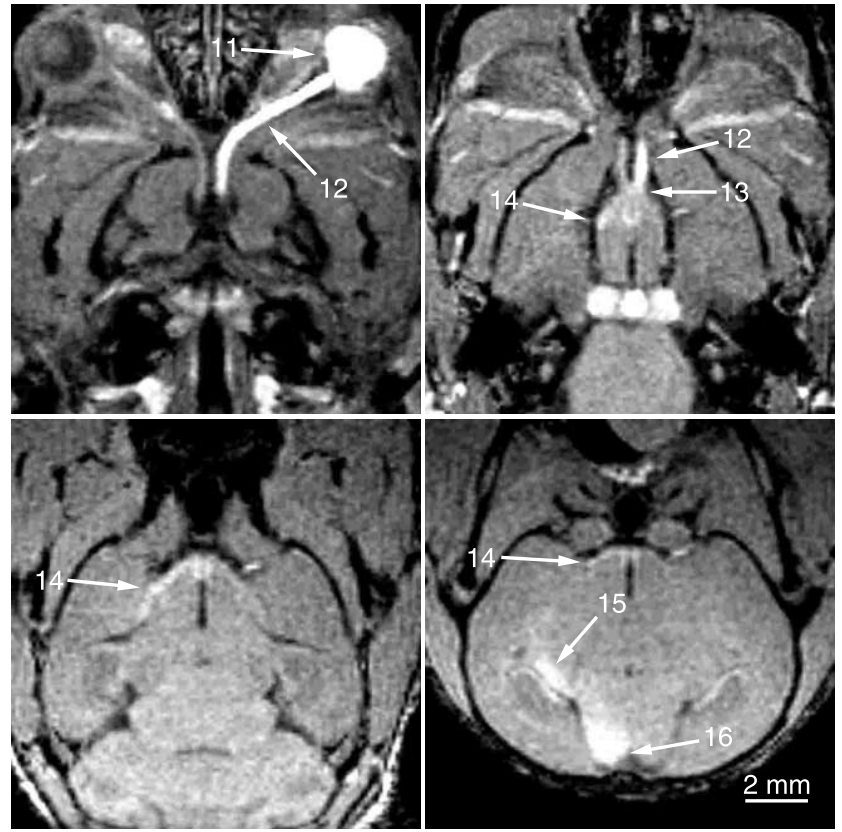

Fig. 6. High-resolution 3D FLASH MRI (for parameters see Table 1) of a mouse brain in vivo (NMRI) demonstrating signal enhancement of the visual pathway $20 \mathrm{~h}$ after injection of $\mathrm{Mn}^{2+}$ into the left eye. The neuroaxonal tract involves the following structures: (11) left retina, (12) left optic nerve, (13) optic chiasm, (14) right optic tract, (15) right lateral geniculate nucleus, and (16) right superior colliculus.

Kooy RF, Verhoye M, Lemmon V, van der Linden A. Brain studies of mouse models for neurogenetic disorders using in vivo magnetic resonance imaging (MRI). Eur J Hum Genet 2001;9:153-9.

Kornguth S, Anderson M, Markley JL, Shedlovsky A. Near-microscopic magnetic resonance imaging of the brains of the phenylalanine hydroxylase-deficient mice, normal littermates, and of normal BALB/c mice at 9.4 Telsa. Neuroimage 1994;1:220-9.

Munasinghe JP, Gresham GA, Carpenter TA, Hall LD. Magnetic resonance imaging of the normal mouse brain: comparison with histologic sections. Lab Anim Sci 1995;45:674-9.

Natt O, Watanabe T, Boretius S, Michaelis T, Frahm J. In vivo highresolution 3D MRI of mouse brain at low field (2.35 T). Proc Intl Soc Mag Reson Med 2002;10:1253.

Rosen GD, Williams AG, Capra JA, Connolly MT, Cruz B, Lu L, Airey DC, Kulkarni K, Williams RW. The mouse brain library, Int Mouse Genome Conf. @ www.mbl.org. 2000;14:166.

Smith BR, Johnson GA, Groman EV, Linney E. Magnetic resonance microscopy of mouse embryos. Proc Natl Acad Sci USA 1994;91:3530-3.

Watanabe T, Michaelis T, Frahm J. Mapping of retinal projections in the living rat using high-resolution 3D gradient-echo MRI with $\mathrm{Mn}^{2+}$-induced contrast. Magn Reson Med 2001;46:424-9.

Xu S, Jordan EK, Li W, Yang Y, Chesnick SA, Webster H, Brocke S, Quigley L, McFarland HF, Frank JA. In vivo three-dimensional MR microscopy of mice and chronic relapsing experimental autoimmune encephalomyelitis after treatment with insulin-like growth factor-I. Am J Neuroradiol 1998;19:653-8.

Yang QX, Smith MB, Briggs RW, Rycyna RE. Microimaging at 14 Tesla using GESEPI for removal of magnetic artifacts in $T_{2}^{*}$ weighted image contrast. J Magn Reson 1999;141:1-6. 